\title{
Clinical delineation of myasthenia gravis in the Kingdom of Bahrain
}

\author{
Mohamed F. Binfalah, MD, Hussein H. Alhafnawi, BSc, Ahmed A. Jaradat, PhD, Eslam Shosha, MD, Ali J. Alhilly, MD, Firas \\ K. Al Nidawi, MD, Mariam M. Alhammadi, MD, Moiz O. Bakhiet, MD, Fatema M. Abdulla, MD.
}

\begin{abstract}

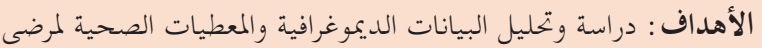

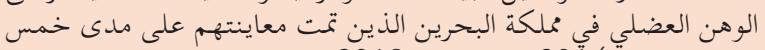

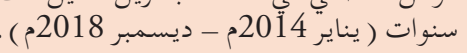

المنهجية : كانت هذه دراسة استعادية قائمة على الملاحظة أجريت في 3

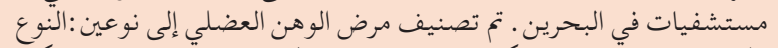

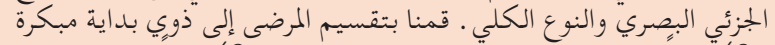

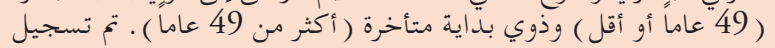

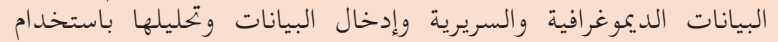

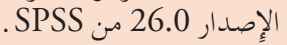

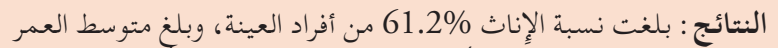

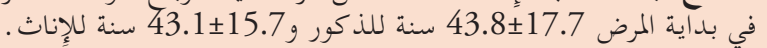

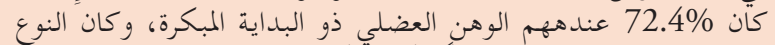

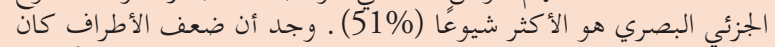

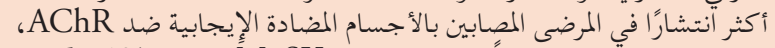

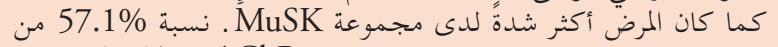

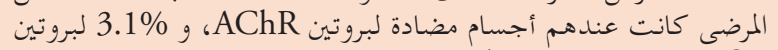
ظuSK

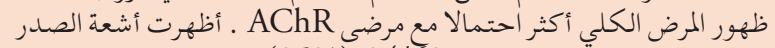

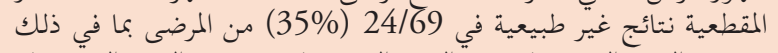

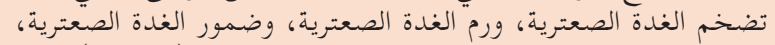

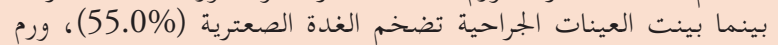

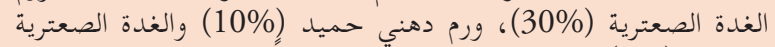
الطبيعية (5\%) . كانت نتائج العلاج جيدة (30\%) جداً في أغلب الحمالات .

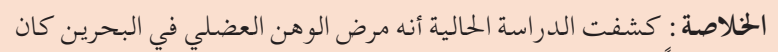

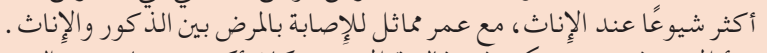

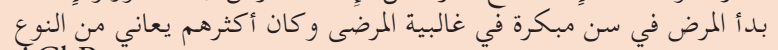

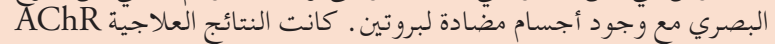

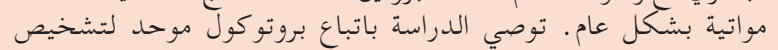
وعلاج مرض الوهن العضلي تولي
\end{abstract}

Objectives: To report demographic and clinical data on 98 myasthenia gravis (MG) patients, seen over 5 years (January 2014-December 2018).

Methods: This was a retrospective, observational cohort study carried out at 3 hospitals in Bahrain. MG was classified into ocular or generalized types. We subdivided MG into early-onset (EOMG, $\leq 49$ years) or late-onset (LOMG, > 49 years). Demographic and clinical data were recorded. The data was entered and analyzed using SPSS version 26.0.

Results: $61.2 \%$ were females. The mean age at onset was $43.8 \pm 17.7$ years in males and $43.1 \pm 15.7$ years in females. $72.4 \%$ had EOMG. A pure ocular presentation was most common (51\%). Limb weakness was more prevalent in AChR-positive patients. The MuSK group had more severe presentation. $57.1 \%$ of patients were AChR-positive, 3.1\% MuSK-positive, and $39.8 \%$ double-seronegative. Generalized disease onset was more likely with AChR. Abnormal CT chest was seen in 24/69 (35\%) including thymic hyperplasia, thymoma, and thymic atrophy. Pathology findings were thymic hyperplasia $(55.0 \%)$, thymoma (30\%), thymolipoma (10\%), and normal thymus $(5 \%)$. Treatment outcomes were favorable.

Conclusion: The present study revealed that MG was more common in females, with similar age at onset between males and females. The majority of patients had EOMG with ocular disease and AChR positivity. The clinical outcomes were favorable. Following a standardized protocol for MG diagnosis and workup is recommended.

Neurosciences 2022; Vol. 27 (1): 16-23 doi: 10.17712/nsj.2022.1.20210096

From Department of Neurology (Binfalah), Department of Family and Community Medicine (Jaradat), Clinical Research Coordinator (Alhafnawi), Aljawhara Center (Bakhiet), Department of Molecular Medicine, Arabian Gulf University, Manama, and from the Neurology and Internal Medicine (Alhilly), Bahrain Defense Force Hospital, West Riffa, from Department of Internal Medicine (Al Nidawi, Alhammadi), King Hamad University Hospital, Busaiteen, from Department of Neurosciences (Abdulla), Salmanya Medical Complex, Ministry of Health, Juffair, Kingdom of Bahrain, and from the Department of Medicine (Shosha), Neurology Division, McMaster University, Hamilton, Canada.

Received 25th July 2021. Accepted 11th October 2021.

Address correspondence and reprint request to: Dr. Mohamed F. Binfalah, University Medical Center, King Abdullah Medical City, Adliya, Kingdom of Bahrain. E-mail: mfalahmd@gmail.com ORCID ID: https://orcid.org/0000-0001-5053-8851 
$M$ yasthenia gravis $(\mathrm{MG})$ is an autoimmune disease of neuromuscular transmission, characterized by fatigable muscle weakness, ${ }^{1}$ mediated by an antibody attack on components of the postsynaptic membrane of the neuromuscular junction, such as the acetylcholine receptor (AChR), muscle-specific tyrosine kinase $(\mathrm{MuSK})$ and lipoprotein receptor-related protein 4 (LRP4)., ${ }^{2,3}$ This may result in various distribution of muscle weakness among different patients, including ocular and limb weakness, bulbar involvement, and sometimes respiratory failure, as well as various degrees of severity. ${ }^{2,4,5}$ The MG is the major disorder that affects the neuromuscular junction, while other rarer syndromes include the Lambert-Eaton myasthenic syndrome and botulism, both affecting the presynaptic neuromuscular junction. ${ }^{4}$ The Myasthenia Gravis Foundation of America (MGFA) clinical classification (class I ocular, class II mild generalized weakness, class III moderate generalized weakness, class IV severe weakness, and class $\mathrm{V}$ needing intubation) is used to identify subgroups of MG patients sharing certain clinical features or disease severity, which may help predict disease prognosis and treatment outcome. ${ }^{6}$

Based on serum antibody profile and clinical phenotypes, MG can be classified into seven subgroups: early-onset MG (EOMG), late-onset MG (LOMG), thymoma-associated MG, MuSK MG, LRP4 MG, ocular MG, and seronegative (SN) generalized MG. ${ }^{4,5,7}$ This classification is clinically useful, as there are important differences in terms of clinical features, thymus abnormalities, treatment outcome, and drug adverse events among different phenotypes. ${ }^{2,5}$ For example, Deymeer et $\mathrm{al}^{5}$ compared the clinical characteristics of 32 anti-MuSK MG, 161 anti-AChR MG and 33 SN MG patients. The MuSK MG group had more bulbar involvement and more severe disease with crises, while the $\mathrm{SN}$ group had a milder disease with a good outcome and lower percentage of steroid maintenance doses and use of other immunosuppressants such as azathioprine. Females predominated in all 3 groups.

In general, the prognosis of MG is good, due to early diagnosis and availability of symptomatic treatment, and the use of effective immunosuppressants and other supportive measures. ${ }^{4}$ The aim of our study was to retrospectively gather data on the clinical characteristics of MG patients in the Kingdom of Bahrain, and compare it with regional and international data.

Disclosure. Authors have no conflict of interests, and the work was not supported or funded by any drug company.
Methods. This was a retrospective, observational cohort study. We reviewed the medical records of all patients with MG seen at 3 government and university hospitals (University Medical Center of King Abdullah Medical City, Salmanya Medical Complex, King Hamad University Hospital) in the Kingdom of Bahrain from January 2014 to December 2018. The inclusion criteria were age 15 years and above, typical clinical presentation of MG, a documented neurological examination of fatigable muscle weakness, and at least one of the following:

- Positive nerve conduction study with $3 \mathrm{~Hz}$ repetitive stimulation revealing $10 \%$ or more motor amplitude decrement.

- Improvement of weakness with edrophonium test.

- Positive serology for at least one of the following antibodies: AChR, MuSK, and LRP4.

Patients with an ultimately different neuromuscular diagnosis during follow-up were excluded. We also excluded patients with missing critical clinical information. The study was approved by the local ethical committee at each participating center.

We collected demographic data, presenting MG symptoms, serology profile, electrophysiology results, radiological studies, pathology reports, treatment received, and outcome at the last follow-up visit. The MG was classified into 2 types: pure ocular (corresponding to MGFA class I), or generalized (MGFA classes II, III, IV, V). We also sub-classified patients into early-onset $(\mathrm{EOMG}, \leq 49$ years age) or late-onset (LOMG, $>49$ years age) disease. The treatment outcomes at the last follow-up visit were divided into three categories, adopted from the MGFA postintervention status classification: ${ }^{6}$ remission (including complete or pharmacologic remissions), improvement (minimal manifestations), or worsening.

The data was entered and analyzed using Statistical Package of Social Science, version 26.0 (IBM SPSS, Armonk, NY, USA). Qualitative variables were presented as counts and percentages. Quantitative variables were presented as means and standard deviations. Two independent samples t-test was used to compare the mean differences between groups. The Chi-square test was used to measure the association and compare proportions. A $p$-value $<0.05$ was considered as a cutoff point of statistical significance.

Results. Tables 1, 2, and 3 summarize the study demographic and clinical data. Table 1 shows the demographic and clinical characteristics of the study population. Ninety-eight patients were included in the study. Sixty (61.2\%) were females (M:F ratio=1:1.5). The mean age of onset \pm standard deviation (SD) was 
Table 1 - Comparison of clinical features between early-onset vs. late-onset MG, all patients.

\begin{tabular}{|c|c|c|c|c|}
\hline Variables & $\operatorname{EOMG}(\mathrm{n}=71,72.4 \%)$ & $\begin{array}{l}\text { LOMG }(\mathrm{n}=27,27.6 \%) \\
\text { n }(\%)\end{array}$ & Total $(n=98)$ & $P$-value \\
\hline Gender & & & & 0.495 \\
\hline Male & $29(29.6)$ & $9(9.2)$ & $38(38.8)$ & \\
\hline Female & $42(42.8)$ & $18(18.4)$ & $60(61.2)$ & \\
\hline MG classification at onset & & & & 0.209 \\
\hline Pure Ocular & $39(39.8)$ & $11(11.2)$ & $50(51.0)$ & \\
\hline Generalized & $32(32.7)$ & $16(16.3)$ & $48(49.0)$ & \\
\hline \multicolumn{5}{|l|}{ Symptoms at onset } \\
\hline Ocular & $60(61.2)$ & $25(25.5)$ & $85(86.7)$ & 0.292 \\
\hline Fatigability & $46(46.9)$ & $14(14.3)$ & $60(61.2)$ & 0.240 \\
\hline Fluctuation & $34(34.7)$ & $10(10.2)$ & $44(44.9)$ & 0.335 \\
\hline Bulbar & $16(16.3)$ & $11(11.2)$ & $27(27.5)$ & 0.072 \\
\hline Facial weakness & $13(13.3)$ & $6(6.1)$ & $19(19.4)$ & 0.662 \\
\hline Respiratory symptoms/failure & $6(6.1)$ & $2(2.0)$ & $8(8.1)$ & 0.866 \\
\hline Neck weakness & $20(20.4)$ & $6(6.1)$ & $26(25.6)$ & 0.551 \\
\hline Limb weakness & $21(21.4)$ & $8(8.2)$ & $29(29.6)$ & 0.996 \\
\hline \multicolumn{5}{|l|}{ Antibody status } \\
\hline AChR & $38(38.8)$ & $18(18.4)$ & $56(57.1)$ & \\
\hline MuSK & $2(2.0)$ & $1(1.0)$ & $3(3.1)$ & 0.447 \\
\hline SN & $31(31.6)$ & $8(8.2)$ & $39(39.8)$ & \\
\hline \multicolumn{5}{|l|}{$N C S(R N S)(n=66)$} \\
\hline Positive & $34(51.5)$ & $12(18.2)$ & $46(69.7)$ & 0.596 \\
\hline Negative & $16(24.2)$ & $4(6.1)$ & $20(30.3)$ & \\
\hline \multicolumn{5}{|l|}{$C T$ scan of the chest $(n=69)$} \\
\hline Normal & $33(47.8)$ & $12(17.4)$ & $45(65.2)$ & 0.592 \\
\hline Abnormal $(n=24)$ & $19(27.5)$ & $5(7.2)$ & $24(34.8)$ & \\
\hline Thymic hyperplasia & $12(48.0)$ & $1(4.0)$ & $13(54.2)$ & \\
\hline Thymoma & $5(20.0)$ & $3(12.0)$ & $8(33.3)$ & \\
\hline Thymic atrophy & $2(8.0)$ & $1(4.0)$ & $3(12.5)$ & \\
\hline \multicolumn{5}{|l|}{ Thymus pathology $(n=20)$} \\
\hline Thymic hyperplasia & $8(40.0)$ & $3(15.0)$ & $11(55.0)$ & \\
\hline Thymoma & $4(20.0)$ & $2(10.0)$ & $6(30.0)$ & 0.740 \\
\hline Thymolipoma & $2(10.0)$ & 0 & $2(10.0)$ & \\
\hline Normal thymus & $1(5.0)$ & 0 & $1(5.0)$ & \\
\hline \multicolumn{5}{|l|}{ Treatment outcome $(n=58)$} \\
\hline Remission & $7(12.1)$ & 0 & $7(12.1)$ & \\
\hline Improvement & $27(46.6)$ & $15(25.9)$ & $42(72.4)$ & 0.165 \\
\hline Worsening & $6(10.3)$ & $3(5.2)$ & $9(15.5)$ & \\
\hline $\begin{array}{r}\text { AChR - acetylcholine recep } \\
\text { LOMG - late onset myasther } \\
\text { NCS - nerve }\end{array}$ & $\begin{array}{l}\text { CT - computed tomogr } \\
\text { gravis (> } 49 \text { years), MG - } \\
\text { duction studies, RNS }-\mathrm{r}\end{array}$ & $\begin{array}{l}\text { EOMG - early onset } \\
\text { asthenia gravis, MuSK } \\
\text { tive nerve stimulation, } 5\end{array}$ & $\begin{array}{l}\text { henia gravis } \\
\text { cle specific ty } \\
\text { eronegative }\end{array}$ & $\begin{array}{l}9 \text { years), } \\
\text { ne kinase, }\end{array}$ \\
\hline
\end{tabular}

$43.8 \pm 17.7$ years in males and $43.1 \pm 15.7$ years in females.

The EOMG was seen in $71 / 98$ (72.4\%), and $27 / 98$ (27.6\%) had LOMG (Table 1). At disease diagnosis, 50/98 (51\%) had pure ocular MG, and 48/98 (49\%) had a generalized disease. There was no statistically significant association between MG age at onset (EOMG or LOMG) with gender $(p=0.495)$ nor with MG classification (pure ocular or generalized, $p=0.209$ ).

Table 1 demonstrates the prevalence of initial presenting symptoms (the same patient may have more than one symptom). The most common symptoms 
Table 2 - Comparison between AChR-positive and double-seronegative MG (excluding MuSK MG patients ${ }^{\dagger}$ ).

\begin{tabular}{|c|c|c|c|c|}
\hline Variables & AChR MG $(\mathrm{n}=56,58.9 \%)$ & $\begin{array}{l}\text { SN MG (n =39, 41.1\%) } \\
\text { n (\%) }\end{array}$ & Total $(\mathrm{n}=95)$ & $P$-value \\
\hline Gender & & $\mathrm{n}(\%)$ & & \\
\hline Male & $23(24.2)$ & $14(14.7)$ & $37(38.9)$ & 0.611 \\
\hline Female & $33(34.7)$ & $25(26.3)$ & $58(61.1)$ & \\
\hline \multicolumn{5}{|l|}{ Onset } \\
\hline EOMG & $38(40.0)$ & $31(32.6)$ & 69 (72.6) & 0.211 \\
\hline LOMG & $18(16.8)$ & $8(10.5)$ & $26(27.3)$ & \\
\hline \multicolumn{5}{|l|}{ MG types at onset } \\
\hline Ocular & $25(26.3)$ & $25(26.3)$ & $50(52.6)$ & 0.062 \\
\hline Generalized & $31(32.6)$ & $14(14.7)$ & $45(47.4)$ & \\
\hline \multicolumn{5}{|l|}{ Symptoms at onset } \\
\hline Ocular & 49 (51.6) & $33(34.7)$ & $82(86.3)$ & 0.687 \\
\hline Fatigability & $38(40.0)$ & $20(21.1)$ & $58(61.1)$ & 0.103 \\
\hline Fluctuation & $26(27.4)$ & $16(16.8)$ & $42(44.2)$ & 0.602 \\
\hline Bulbar & $19(20)$ & $7(7.4)$ & $26(27.4)$ & 0.086 \\
\hline Facial weakness & $14(14.7)$ & $4(4.2)$ & $18(18.9)$ & 0.071 \\
\hline Respiratory symptoms/failure & $6(6.3)$ & $2(2.1)$ & $8(8.4)$ & 0.335 \\
\hline Neck weakness & $18(18.9)$ & $7(7.4)$ & $25(26.3)$ & 0.122 \\
\hline Limb weakness & $22(23.1)$ & $5(5.3)$ & $27(28.4)$ & 0.005 \\
\hline \multicolumn{5}{|l|}{$N C S(R N S)(n=63)$} \\
\hline Positive & $30(47.6)$ & $14(22.2)$ & $44(68.2)$ & 0.007 \\
\hline Negative & $6(9.5)$ & $13(20.6)$ & $19(31.8)$ & \\
\hline \multicolumn{5}{|l|}{ Chest CT $(n=67)$} \\
\hline Normal & $26(47.6)$ & $18(22.2)$ & $44(65.7)$ & 0.006 \\
\hline Abnormal & $21(31.3)$ & $02(3.0)$ & $23(34.3)$ & \\
\hline Thymic hyperplasia & $10(15.2)$ & $2(3)$ & $12(18.2)$ & \\
\hline Thymoma & $8(12.1)$ & 0 & $8(12.1)$ & \\
\hline Thymic atrophy & $3(4.5)$ & 0 & $3(4.5)$ & \\
\hline \multicolumn{5}{|l|}{ Treatment outcome $(n=55)$} \\
\hline Remission & $4(7.3)$ & $2(3.6)$ & $6(10.9)$ & \\
\hline Improvement & $28(50.9)$ & $12(21.8)$ & $40(72.7)$ & 0.972 \\
\hline Worsening & $6(10.9)$ & $3(5.5)$ & $9(16.4)$ & \\
\hline $\begin{array}{l}\text { AChR - acetylcholine recepto } \\
\text { myasthenia gravis (> } 49 \text { years), } \\
\text { - repetitive nerve stimulation, }\end{array}$ & $\begin{array}{l}\mathrm{T} \text { - computed tomography, EC } \\
\mathrm{G} \text { - myasthenia gravis, MuSK - } \\
\text { - seronegative, }{ }^{\dagger} 3 \mathrm{MuSK} \text { patien } \\
\text { disease, } 2 \text { with normal CT ch }\end{array}$ & $\begin{array}{l}\text { - early onset myasthenia gr: } \\
\text { e specific tyrosine kinase, N } \\
\text { emales and } 1 \text { male, } 2 \text { with } \mathrm{F} \\
\text { id } 1 \text { with thymic hyperplasi }\end{array}$ & $\begin{array}{l}49 \text { years), LOM } \\
\text { nerve conductio } \\
\text { and } 1 \text { LOMG }\end{array}$ & $\begin{array}{l}\text { late onset } \\
\text { udies, RNS } \\
\text { generalizec }\end{array}$ \\
\hline
\end{tabular}

were ocular (diplopia and/or ptosis) in $86.7 \%$, followed by muscle fatigability $(61.2 \%)$, and fluctuation of symptoms (44.9\%). Respiratory symptoms and/or respiratory failure were seen in $8.1 \%$. There was no statistically significant association between any of the initial presenting symptoms and early-onset or late-onset disease.

Table 2 compares the AChR-positive MG with double seronegative MG patients (the MuSK group was reported separately due to the small number). With regards to the initial symptoms and serostatus, AChR- positivity is more common among patients who reported limb weakness $(22 / 27,81.4 \%, p=0.005)$. Furthermore AChR-positivity is more frequent among patients who reported bulbar symptoms $(19 / 26,73.1 \%, p=0.086)$, and facial weakness $(14 / 18,77.8 \%, p=0.071)$, but this was not statistically significant.

Serum AChR antibodies were present in 56/98 patients $(57.1 \%)$, while $3 / 98(3.1 \%)$ were positive for MuSK antibodies (Table 1). The rest (39/98, 39.8\%) were double-seronegative. No LRP4-positive patients were identified. $55.4 \%$ of the AChR-positive patients 
Table 3 - Treatments used in MG patients throughout the disease course, $\mathrm{n}=98$

\begin{tabular}{lc}
\hline Treatment & Patients, $\mathbf{n}(\%)$ \\
\hline Pyridostigmine & $88(89.8)$ \\
Prednisone & $63(64.3)$ \\
Mycophenolate Mofetil & $15(15.3)$ \\
Azathioprine & $27(27.6)$ \\
IVIG & $23(23.5)$ \\
Rituximab & $2(2)$ \\
\hline
\end{tabular}

(31/56) presented with generalized disease (MGFA classes II, III, IV, and V) at onset (Table 2), while the majority of the double-seronegative group (25/39, 64.1\%) had milder disease presentation with only ocular symptoms (MGFA I). In both the AChRpositive and the double-seronegative groups, there was no statistically significant correlation between serology status and MG types (ocular or generalized) at onset $(p=0.062)$.

Only 3/98 (3.1\%) patients of our cohort were MuSK-positive ( 2 females, one male). One presented with bulbar weakness, while the other 2 presented with more generalized symptoms including bulbar and limb weakness; no respiratory symptoms were seen in any of these patients. Two patients had EOMG and one LOMG. The CT chest was normal in 2 patients, while one patient had thymic hyperplasia and underwent thymectomy.

Electrophysiological studies were available for $66 / 98$ patients, out of whom $46(69.7 \%)$ showed positive decrement response on $3 \mathrm{HZ}$ repetitive nerve stimulation (Table 1). There was no correlation between abnormal repetitive nerve stimulation findings and EOMG or LOMG $(p=0.596)$. As shown in Table 2, a positive decrement was more commonly seen in the AChR positive group $(30 / 44,68.2 \%)$ compared to the double-seronegative group $(14 / 44,31.8 \%)$ patients $(p=0.007)$.

The results of the chest computed tomography (CT) scan were available in 69/98 (70\%) patients (Table 1). Thymic abnormalities were seen in 24/69 (35\%), namely thymic hyperplasia $(13 / 24,54.2 \%)$, thymoma $(8 / 24$, $33.3 \%)$, and thymic atrophy $(3 / 24,12.5 \%)$. Chest CT findings were associated with serology status (Table 2), as abnormal findings were more prevalent among patients with AChR-positivity $(21 / 23,91.3 \%)$ compared to the double-seronegative $(2 / 23,8.7 \%)$ patients $(p=0.006)$. Likewise, an abnormal chest CT was more common in patients with more generalized symptoms, specifically neck weakness $(p=0.047)$ and limb weakness $(p=0.001)$.
Thymectomy (Table 1) was carried out in 20/98 patients ( 7 males, 13 females), with pathology revealing thymic hyperplasia in $11(55.0 \%)$, thymoma in 6 $(30.0 \%)$, thymolipoma in $2(10.0 \%)$, and normal thymic tissue in $1(5.0 \%) .75 \%$ of thymectomy patients had EOMG and $85 \%$ were AChR positive $(p=0.023)$. One patient was MuSK-positive, and 2 patients were double-seronegative. All patients had a generalized disease.

The medications used in our cohort are shown in Table 3. Most patients (90\%) were treated with acetylcholinesterase inhibitors (namely pyridostigmine), followed by prednisone (64.3\%). Other treatments included intravenous immunoglobulin (IVIG), mycophenolate mofetil, and azathioprine, while rituximab was used in only 2 patients. The data on the use of plasmapheresis were not available, although in Bahrain it is being used frequently in patients with myasthenic crises.

The treatment outcomes at the last follow-up visit were evaluated for $58 / 98$ patients (Table 1). $7 / 58$ (12.1\%) went into remission, $42 / 58(72.4 \%)$ reported significant improvement, and 9/58 (15.5\%) reported disease worsening. $12.1 \%$ of EOMG patients went into complete remission, while none had remission in the LOMG. Regarding serostatus (Table 2), there was no correlation with treatment outcome $(p=0.972)$. All patients in the post-thymectomy group (all pathology types) had significant improvement or went into remission.

Discussion. This study from the Kingdom of Bahrain reports the clinical characteristics and profile of patients with MG seen over 5 years and compares it with regional and international cohorts.

Many cohorts and registries of MG patients have been published from different countries worldwide..$^{8-12}$ For example, Blum et $\mathrm{al}^{11}$ presented a communitybased survey of 165 Australian MG patients. EOMG patients (defined as less than 40 years of age) were more frequently females, while males were more frequently affected in the LOMG. Other coexisting autoimmune disorders were reported in 54\% of the study population. The majority of patients were AChR-positive, and a small number had MuSK MG. The most common initial symptoms were weak eyelids $(67 \%)$ and diplopia (60\%), followed by arm (55\%) and leg (52\%) weakness.

Another Canadian study ${ }^{10}$ reported the epidemiology of MG in Ontario, Canada between 1996-2013. The MG prevalence showed a steady increase from 16.6 per 100,000 population in 1996 , to 32.0 per 100,000 population in 2013, while the crude incidence rates 
remained stable over the same period (2.7 and 2.8 per 100,000 population in 1996 and 2013, respectively). The incidence rates were higher in females less than 50 years of age and in males more than 50 years of age.

There have been few published reports on the clinical features and demographics of MG patients from the Arabian Gulf region. ${ }^{13-16}$ A study from Saudi Arabia ${ }^{13}$ retrospectively reported the clinical data on $104 \mathrm{MG}$ patients followed over a mean period of 7.2 years at a single institution. The mean age of onset was 22.5 years in females and 28.2 in males, with peaks in the second and third decays among females and third and fourth decades among males. At disease diagnosis, the majority of patients had moderate generalized weakness equivalent to MGFA class III. Most of the patients were positive for AChR antibodies (73\%). The prevalence of MuSK MG was not reported. After medical treatment, with or without thymectomy, most patients achieved either complete stable remission, pharmacologic remission, or had minimal manifestations, while 35\% remained either unchanged or worsened.

Another report from $\mathrm{Oman}^{14}$ followed 50 consecutive $\mathrm{MG}$ patients for a mean period of three years at a major university hospital. The mean age at presentation was 27 years (range 5-63 years), with a 2:3 male to female ratio. Six patients $(12 \%)$ presented with ocular MG, while the rest had a generalized disease at onset. Bulbar and respiratory involvements were more common in this series compared with western data. Thymectomy was done in 29 patients, with 8 of them achieving complete stable remission for 2 years.

In our cohort, female patients predominated, compromising $61.2 \%$, with a male: female ratio of $1: 1.5$, which is similar to other cohorts from the Arabian Gulf, ${ }^{13-16}$ and internationally, ${ }^{9-12,17}$ where, for instance, females represented $62 \%$ and $58 \%$ respectively in 2 cohorts from Saudi Arabia, ${ }^{13,15}$ and 54\% in an Australian study. ${ }^{11}$ We found that the mean age of onset of MG was similar between males and females (43 years). This is in comparison to studies from different regions that revealed a bimodal age of onset with 2 peaks, in young females and older males. ${ }^{10,11,13,15}$ However, a Korean study found that the mean age of onset was almost similar between the 2 genders $(32.5 \pm 19.4$ years in males and $30.8 \pm 17.3$ years in females), similar to our findings. ${ }^{18}$ We suspect that the exclusion of juvenile MG cases in our cohort, which were included in other studies, might have affected the age of onset.

The majority of patients had EOMG, which is in line with several regional studies. ${ }^{13,15}$ We did not observe any gender predominance in either the EOMG or LOMG patients $(p=0.495)$.
The most common disease presentation was ocular (MGFA I), seen in more than half of patients, which is significantly different from cohorts reported from Oman, ${ }^{14}$ Saudi Arabia, ${ }^{13,15}$ and UAE, ${ }^{16}$ where the majority of these cohorts' patients had generalized MG at disease onset. This might be partly due to the ability of patients to access neurology services in Bahrain relatively early in their disease course, as well as improved knowledge of the disease among physicians and neurologists, allowing for detection of early mild cases, although other environmental, ethnic, or genetic factors might play a role as well, both in disease susceptibility and severity. ${ }^{1,19}$ There was no correlation between MG classification at onset (ocular or generalized) and age at presentation $(p=0.209)$.

With regards to the serology findings, the majority of patients were positive for AChR antibodies, while the overall MuSK-positive cases were only 3.1\%. However, among the patients who were AChR-negative, the MuSK prevalence was $7.3 \%$. It is known that the incidence of MuSK MG varies significantly between different countries, being more common in the Mediterranean European regions and East Asia northern regions., ${ }^{40-22}$ There is also a high incidence reported in African Americans than in Caucasians. ${ }^{22}$ These differences in incidence are thought to be due to certain genetic factors. ${ }^{4}$ We also cannot ascertain whether this test was ordered for all patients with negative AChR antibodies, which potentially may have contributed to the low MuSK-positive cases.

We found a significant correlation between serology findings and presenting symptoms, with more generalized symptoms at onset in the AChR-positive and MuSK-positive patients, while the doubleseronegative patients were more likely to present with mild ocular MG (MGFA I). It is well-known that only $50 \%$ of ocular MG patients have detectable antibodies, ${ }^{4}$ which explains the higher percentage of seronegativity in our ocular MG group. However, since this was a retrospective study, we suspect that some of these patients might develop a generalized disease over time and their antibody status might change as well. ${ }^{4}$

Regarding abnormalities on repetitive nerve conduction studies, most patients had abnormal findings, but a significant decrement was more likely to be found in AChR-positive patients $(p=0.007)$ and those with generalized disease $(p=0.01)$.

All patients were put on medications, either a single agent or a combination regimen, e.g. pyridostigmine, prednisolone, mycophenolate mofetil, or azathioprine. IVIG was used mostly in patients with more severe symptoms, in preparation for surgery, or MG crisis. Unfortunately, although plasmapheresis is commonly 
used in practice, the data on its use was not found. Rituximab has been reported to be associated with favorable outcomes particularly in MuSK MG, ${ }^{23-25}$ but it was used in only two patients, probably because it was not available in some of the study centers.

Several studies, ${ }^{26,27}$ and guidelines ${ }^{28}$ have concluded that thymectomy in patients with non-thymomatous MG improves clinical outcomes over the long term, with resultant minimization of the use of immunotherapy and fewer exacerbations. It is usually performed in patients below 60 years of age with AChR-positive disease and generalized symptoms. ${ }^{3}$ In thymomatous MG, all patients, with rare exceptions, should undergo thymectomy to remove the tumor. ${ }^{28}$

In our cohort, the vast majority of thymectomies were carried out following international consensus guidance for $\mathrm{MG},{ }^{26,28}$ either for suspected thymomas or thymic hyperplasia in patients with generalized, AChR-positive early-onset MG. Most of the nonthymomatous patients undergoing surgery in our study had EOMG with generalized disease and thymic hyperplasia on histology, in line with the findings from previously reported regional cohorts ${ }^{13}$ revealing more than two thirds with thymic hyperplasia on pathology. We found a relatively high proportion of thymomas $(30 \%)$, but due to the relatively small number of thymectomy procedures performed, we cannot make a reliable conclusion on this finding.

The finding of thymolipomas in 2 patients is particularly interesting. These are uncommon, benign tumors that represent $2-9 \%$ of all thymic masses..$^{29,30}$ This benign entity has been reported to be associated with MG as well as other autoimmune conditions, e.g. Grave's disease, aplastic anemia, and lichen planus, among others. ${ }^{31,32}$ Thymolipomas are more common in younger patients, usually asymptomatic, seen as large mediastinal tumors on chest radiographs. ${ }^{31}$ In a study comparing the surgical outcome of thymolipomatous MG with thymomatous MG and non-thymomatous MG, the results were similar between patients with thymolipomatous and those with non-thymomatous MG. ${ }^{33}$ The rate of stable remission was higher in the thymolipomatous $(41.7 \%)$ and non-thymomatous $(42.3 \%)$ groups compared to the thymomatous patients (28.8\%).

We found an overall favorable prognosis, regardless of age, disease classification at onset, or antibody status, with most patients achieving either complete remission or significant clinical improvement. The same was true in the thymectomized group as well, irrespective of the pathology type. This is likely related to several factors, such as early detection of mild disease and the use of standard immunosuppressants, and performing thymectomy when clinically indicated.
Our study has several limitations. Some MG patients might not be accounted for as they were not seen at our participating centers. We also excluded young patients $<15$ years of age, as their medical charts were not easily accessible to us, and other clinical entities (e.g. congenital MG syndromes) can be difficult to differentiate from acquired $M G$ due to similar presentation and seronegativity. ${ }^{34,35}$ This exclusion may have potentially introduced a sampling bias in terms of MG age at onset, which classically has a bimodal distribution of age (younger females and older males), while we found a nearly similar mean age in both males and females. Due to the retrospective design of our study, some important clinical, serology, and radiology data could not be traced, limiting a detailed statistical analysis and correlations in some instances. Nevertheless, we could extract enough meaningful and relevant data to better describe the MG cohort in this study.

In conclusion, the present study revealed that in this cohort, MG was more common in females, with similar age at onset between males and females. The majority of patients had EOMG with mild ocular disease presentation and AChR positivity. Symptomatic and immunosuppressants treatment with acetylcholinesterase inhibitors and steroids were commonly used with overall good treatment response. The clinical outcomes were favorable irrespective of disease classification, antibody status, or thymus pathology. Following a standardized protocol for MG diagnosis and workup is advised, and a larger prospective nationwide study, including the juvenile MG cohort, is recommended to validate our findings and shed more light on this uncommon neuromuscular disorder.

Acknowledgement. The authors would like to thank Dr. Adel Medhkour (Director of Clinical Research Center at Arabian Gulf University) for his valuable advice and support during all the stages of this research, and Dr. Jameel Ahmed, consultant Radiologist at King Abdullah Medical City, for reviewing the radiology. We would also like to thank Rasayely English Editing Company for English language editing.

\section{References}

1. Binks S, Vincent A, Palace J. Myasthenia gravis: a clinicalimmunological update. J Neurol 2016; 263: 826-834.

2. Cortés-Vicente E, Álvarez-Velasco R, Segovia S, Paradas C, Casasnovas C, Guerrero-Sola A, et al. Clinical and therapeutic features of myasthenia gravis in adults based on age at onset. Neurology 2020; 94: e1171-e1180.

3. Lindstrom JM, Seybold ME, Lennon VA, Whittingham S, Duane DD. Antibody to acetylcholine receptor in myasthenia gravis. Neurology 1998; 51: 933.2-933-a. 
4. Gilhus NE. Myasthenia Gravis. N Engl J Med 2016; 375: 2570-2581.

5. Deymeer F, Gungor-Tuncer O, Ylmaz V, Parman Y, Serdaroglu P, Ozdemir C, et al. Clinical comparison of anti-MuSK- vs anti-AChR-positive and seronegative myasthenia gravis. Neurology 2007; 68: 609-611.

6. Jaretzki A, Barohn RJ, Ernstoff RM, Kaminski HJ, Keesey JC, Penn AS, et al. Myasthenia gravis: Recommendations for clinical research standards. Ann Thorac Surg 2000; 70: 327-334.

7. Cho EB, Min JH, Lee S, Yoon CW, Seok JM, Cho HJ, et al. Late-onset non-thymomatous myasthenia gravis: Comparison with early-onset and very late-onset myasthenia gravis. Neurol Asia 2017; 22: 123-131.

8. Fulvio B, Mantegazza R. European database for myasthenia gravis: A model for an international disease registry. Neurology 2014; 83: 189-191.

9. Hong Y, Li HF, Skeie GO, Romi F, Hao HJ, Zhang X, et al. Autoantibody profile and clinical characteristics in a cohort of Chinese adult myasthenia gravis patients. J Neuroimmunol 2016; 298: 51-57.

10. Breiner A, Widdifield J, Katzberg HD, Barnett C, Bril V, Tu K. Epidemiology of myasthenia gravis in Ontario, Canada. Neuromuscul Disord 2016; 26: 41-46.

11. Blum S, Lee D, Gillis D, McEniery DF, Reddel S, McCombe P. Clinical features and impact of myasthenia gravis disease in Australian patients. J Clin Neurosci 2015; 22: 1164-1169.

12. Westerberg E, Punga AR. Epidemiology of Myasthenia Gravis in Sweden 2006-2016. Brain Behav 2020; 10.

13. Al-Moallem MA, Alkali NH, Hakami MA, Zaidan RM. Myasthenia gravis: Presentation and outcome in 104 patients managed in a single institution. Ann Saudi Med 2008; 28 : 341-345.

14. Jacob PC, Tharakan JT, Chand PR, Koul RL, Chacko AP. Clinical profile of myasthenia gravis in the Sultanate of Oman. Saudi Med J 2003; 24: 774-775.

15. Alanazy MH. Clinical features and outcomes of patients with myasthenia gravis. Neurosciences 2019; 24: 176-184.

16. Malik YM, Dar JA, Almadani AA. Role of Myasthenia Gravis Auto-Antibodies as Predictor of Myasthenic Crisis and Clinical Parameters. J Neurol Neurosci 2019; 10: 1-6.

17. Tomschik M, Hilger E, Rath J, Mayer EM, Fahrner M, Cetin $\mathrm{H}$, et al. Subgroup stratification and outcome in recently diagnosed generalized myasthenia gravis. Neurology 2020; 95: e1426-e1436.

18. Lee KW, Kim JS, Lee SH, and MG Study Group. Epidemiological and Clinical Characteristics of Myasthenia Gravis in Korea. J Korean Neurol Assoc 1997; 15: 825-838.

19. Avidan N, Le Panse R, Berrih-Aknin S, Miller A. Genetic basis of myasthenia gravis - A comprehensive review. J Autoimmun 2014; 52: 146-153.

20. Koneczny I, Cossins J, Vincent A. The role of muscle-specific tyrosine kinase (MuSK) and mystery of MuSK myasthenia gravis. J Anat 2014; 224: 29-35.

21. Park KH, Waters P, Woodhall M, Lang B, Smith T, Sung JJ, et al. Myasthenia gravis seronegative for acetylcholine receptor antibodies in South Korea: Autoantibody profiles and clinical features. PLoS One 2018; 13: e0193723.
22. Oh SJ, Morgan MB, Lu L, Hatanaka Y, Hemmi S, Young A, et al. Racial differences in myasthenia gravis in Alabama. Muscle and Nerve 2009; 39: 328-332.

23. Hehir MK, Hobson-Webb LD, Benatar M, Barnett C, Silvestri NJ, Howard JF, et al. Rituximab as treatment for anti-MuSK myasthenia gravis. Neurology 2017; 89: 1069-1077.

24. Tannemaat MR, Verschuuren JJGM. Emerging therapies for autoimmune myasthenia gravis: Towards treatment without corticosteroids. Neuromuscul Disord 2020; 30: 111-119.

25. Guptill JT, Sanders DB, Evoli A. Anti-MuSK antibody Myasthenia gravis: Clinical findings and response to treatment in two large cohorts. Muscle and Nerve 2011; 44: 36-40.

26. Wolfe GI, Kaminski HJ, Aban IB, Minisman G, Kuo H-C, Marx A, et al. Randomized Trial of Thymectomy in Myasthenia Gravis. N Engl J Med 2016; 375: 511-522.

27. Baggi F, Andreetta F, Maggi L, Confalonieri P, Morandi L, Salerno F, et al. Complete stable remission and autoantibody specificity in myasthenia gravis. Neurology 2013; 80: 188-195.

28. Sanders DB, Wolfe GI, Benatar M, Evoli A, Gilhus NE, Illa I, et al. International consensus guidance for management of myasthenia gravis: executive summary. Neurology 2016; 87: 419-425.

29. Goswami A, Baruah AR. Giant thymolipoma: A rare case presentation. Asian Cardiovasc Thorac Ann 2017; 25: 143-145.

30. Rieker RJ, Schirmacher P, Schnabel PA, Moser K, Hoffmann $\mathrm{H}$, Dienemann H, et al. Thymolipoma. A report of nine cases, with emphasis on its association with myasthenia gravis. Surg Today 2010; 40: 132-136.

31. Othman SA, Alfrayyan OY, Alghamdi ZM, Makhdom F, Aljehani Y, Elbawab HY, et al. Thymolipoma association with myasthenia gravis: Case report. Am J Case Rep 2020; 21: e923989-1-e923989-4.

32. Tsukioka T, Inoue K, Iwata T, Mizuguchi S, Morita R, Suehiro S. Thymolipoma associated with myasthenia gravis. Gen Thorac Cardiovasc Surg 2007; 55: 26-28.

33. Huang CS, Li WY, Lee PC, Kao KP, Chou TY, Wu MH, et al. Analysis of outcomes following surgical treatment of thymolipomatous myasthenia gravis: Comparison with thymomatous and non-thymomatous myasthenia gravis. Interact Cardiovasc Thorac Surg 2014; 18: 475-481.

34. Huang X, Li Y, Feng H, Chen P, Liu W. Clinical characteristics of juvenile myasthenia gravis in Southern China. Front Neurol 2018; 9: 1-7.

35. Barraud C, Desguerre I, Barnerias C, Gitiaux C, Boulay C, Chabrol B. Clinical features and evolution of juvenile myasthenia gravis in a French cohort. Muscle and Nerve 2018; 57: 603-609. 